\title{
Tumoricidal effect of calicheamicin immuno-conjugates using a passive targeting strategy
}

\author{
ERWIN R. BOGHAERT ${ }^{1}$, KIRAN KHANDKE ${ }^{1}$, LATHA SRIDHARAN ${ }^{1}$, DOUGLAS ARMELLINO ${ }^{1}$, \\ MAUREEN DOUGHER ${ }^{1}$, JOHN F. DiJOSEPH ${ }^{1}$, ARTHUR KUNZ ${ }^{2}$, PHILIP R. HAMANN ${ }^{2}$, \\ ASHWIN SRIDHARAN ${ }^{1}$, STANLEY JONES $^{1}$, CAROLYN DISCAFANI $^{1}$ and NITIN K. DAMLE ${ }^{1}$ \\ ${ }^{1}$ Wyeth Discovery Oncology; ${ }^{2}$ Wyeth Discovery Chemical and Screening Sciences, Pearl River, NY 10965, USA
}

Received September 29, 2005; Accepted November 7, 2005

\begin{abstract}
Calicheamicin is a potent chemotherapeutic with a low therapeutic index that requires targeting to tumor cells for its use in the clinic. To treat acute myeloid leukemia, calicheamicin has been conjugated to an antibody that recognizes CD33 (gemtuzumab ozogamicin). The application range of this 'active' targeting strategy is limited since it depends on specific antigen expression by tumor cells. This limitation could be reduced by using an antigen-independent 'passive targeting' strategy for calicheamicin. 'Passive targeting' relies on the dysfunctional vasculature of a neoplastic tumor that allows enhanced retention of macromolecules. We studied the efficacy of calicheamicin conjugated to various carrier molecules: i.e. immunoglobulin, albumin or PEGylated $\mathrm{Fc}$ fragments. In nude mice, a conjugate of anti-CD33 and calicheamicin accumulates in human tumor xenografts in the absence of detectable amounts of targeting antigen. Passive targeting provided sufficient accumulation of this conjugate to inhibit tumor growth of 10 different CD33-negative xenograft models. This efficacy depended on the use of an acidlabile linker between antibody and calicheamicin. Substitution of immunoglobulin as a carrier with either albumin or PEGylated Fc reduced or eliminated the efficacy of the conjugate. The results showed that using 'non-specific' immunoglobulin for passive targeting of calicheamicin might be an effective mode of cancer therapy.
\end{abstract}

\section{Introduction}

The low therapeutic index of cytotoxic chemotherapy used for cancer treatment prevents administration of sufficiently high doses necessary to eradicate a tumor. To circumvent this problem, several drug delivery strategies involving macromolecular or particulate carriers have been designed to produce

Correspondence to: Dr Erwin R. Boghaert, Wyeth Research, 401 N. Middletown Rd., Pearl River, NY 10965, USA

E-mail:boghaee@wyeth.com

Key words: drug delivery system, chemotherapy, targeted therapy, carcinoma, calicheamicin cancer selective therapeutics (1). A passive targeting strategy relies on the enhanced permeability and retention effect (EPR) (2) of a tumor. This effect allows accumulation of particles or water-soluble macromolecules in a tumor because of the leakiness of the fenestrated endothelium of its blood vessels combined with inadequate lymphatic drainage. Examples of carriers used for this mode of targeting are liposomes (3), albumin (4), dextran (5) or poly(ethylene glycol) (PEG) polymers (6).

For an active tumor targeting strategy (1), the carrier needs a targeting component that specifically interacts with select cells or cell matrix components in a tumor. Specific antibodies that recognize tumor-associated antigens (TAA) are well studied and clinically used carriers for active tumor targeting. In this regard, we developed a conjugate [gemtuzumab ozogamicin, GO (7)] of the disulfide derivative $\mathrm{N}$-acetyl $\gamma$ calicheamicin dimethyl hydrazide (CalichDMH) and the anti-CD33 antibody, hP67.6. CalichDMH is a semi-synthetic calicheamicin that qualifies as a potent double-stranded DNA-targeting cytotoxic antibiotic (8). GO is indicated for the treatment of acute myeloid leukemia in elderly patients.

Active and passive targeting approaches have historically been pursued as separate strategies. However, there is evidence that antibodies do not necessarily require their target antigen to accumulate in tumor tissue $(9,10)$. We recently observed that GO inhibited growth of CD33-negative human tumor xenografts in nude mice (11). This finding suggested that antibodies not only targeted tumor tissue without requirement of the targeting antigen but also were capable of carrying sufficient amounts of calicheamicin to obtain therapeutic efficacy. Using only passive targeting of immunoglobulin to accumulate a cytotoxic agent in a tumor is rarely acknowledged as a feasible strategy for anticancer therapy.

Currently, we report that passive targeting of calicheamicin by means of immunoglobulin as a carrier causes tumor growth inhibition of a variety of human xenografts in nude mice, suggesting the feasibility of this strategy in cancer patients. Mechanistically, the anti-tumor efficacy of calicheamicin administered as a calicheamicin-immunoglobulin conjugate $(\mathrm{CIgC})$ was related to the sensitivity of the tumor cells to the drug and depended on the use of an acid-labile linker between the drug and the immunoglobulin. Substitution of immunoglobulin as a carrier with either albumin or PEGylated Fc reduced or eliminated the efficacy of the conjugate. 
Table I. CD33-expression on carcinoma and leukemia cells in vitro.

\begin{tabular}{|c|c|c|c|c|c|c|c|}
\hline \multirow[b]{2}{*}{ Cell line designation } & \multirow[b]{2}{*}{ Tissue of origin } & \multicolumn{3}{|c|}{ reMCF hP67.6 ${ }^{\mathrm{a}}$} & \multicolumn{3}{|c|}{ reMCF hP67.6-AcBut-CalichDMH } \\
\hline & & Average & $\mathrm{n}^{\mathrm{b}}$ & $\mathrm{S}^{\mathrm{c}}$ & Average & $\mathrm{n}$ & s \\
\hline N87 & Gastric carcinoma & 1.03 & 8 & 0.23 & 1.22 & 4 & 0.93 \\
\hline НТ29 & Colon carcinoma & 1.02 & 10 & 0.35 & 1.41 & 7 & 1.20 \\
\hline LOVO & Colon carcinoma & 0.83 & 5 & 0.09 & 0.85 & 5 & 0.17 \\
\hline PC14PE6 & $\mathrm{NSCLC}^{\mathrm{e}}$ & 0.97 & 6 & 0.34 & 0.99 & 6 & 0.41 \\
\hline L2987 & NSCLC & 0.94 & 5 & 0.17 & 0.94 & 5 & 0.32 \\
\hline MDAMB435/5T4 & Breast carcinoma & 0.66 & 7 & 0.21 & 0.80 & 6 & 0.13 \\
\hline A431 & Epidermoid carcinoma & 0.93 & 12 & 0.36 & 0.97 & 8 & 0.38 \\
\hline A431/Le ${ }^{y}$ & Epidermoid carcinoma & 0.99 & 5 & 0.61 & 0.97 & 5 & 0.54 \\
\hline LNCaP & Prostate carcinoma & 1.08 & 8 & 0.43 & 0.90 & 8 & 0.42 \\
\hline PC3MM2 & Prostate carcinoma & 3.06 & 6 & 1.57 & 3.05 & 5 & 2.17 \\
\hline KG-1 & Myeloid leukemia & 9.07 & 7 & 6.21 & 7.55 & 6 & 4.08 \\
\hline
\end{tabular}

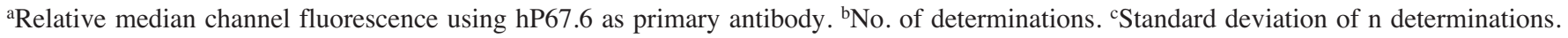
${ }^{\mathrm{d}}$ Relative median channel fluorescence using hP67.6-AcBut-CalichDMH as primary antibody. ${ }^{\mathrm{e}}$ Non-small cell lung carcinoma. Cell lines with reMCF $>4$ are considered CD33-positive.

\section{Materials and methods}

Cells and culturing conditions. N87 (CRL-5822), HT29 (HTB-38), LOVO (CCL-229), A431 (CRL-1555), KG-1 and LNCaP (CRL-1740) were purchased from the American Type Culture Collection (ATCC). Cell lines obtained from ATCC were maintained in culture medium as specified in the ATCC-catalogue. L2987 was a gift from Dr C. Siegall (Seattle Genetics, Bothell, WA). These cells were grown in RPMI1640 supplemented with $10 \%$ FBS, 2 mM gln, $100 \mathrm{IU} / \mathrm{ml}$ penicillin and $100 \mu \mathrm{g} / \mathrm{ml}$ streptomycin (hereafter called pen/ strep) and $0.05 \mathrm{mg} / \mathrm{ml}$ gentamycin. PC14PE6 (12), PC3MM2 and MDAMB435 were obtained from Dr I. Fidler (M.D. Anderson, Houston, TX). PC14PE6 and PC3MM2 were maintained in minimum essential medium supplemented with $10 \% \mathrm{v} / \mathrm{v}$ FBS, $2 \mathrm{mM}$ gln, $1 \mathrm{mM}$ sodium pyruvate, $0.2 \mathrm{mM}$ non-essential amino acids, 2\% MEM vitamin solution, and pen/strep. MDAMB435/5T4 are MDAMB435 cells that were transfected with a plasmid encoding the oncofetal protein, 5T4, and the neomycin resistance marker. These cells were cultured in minimum essential medium with Earle's salts supplemented with $10 \%$ v/v FBS, $2 \mathrm{mM}$ gln, $1 \mathrm{mM}$ sodium pyruvate, $0.2 \mathrm{mM}$ non-essential amino acids, $2 \%$ MEM vitamin solution, and $50 \%$ pen/strep and $1.5 \mathrm{mg} / \mathrm{ml} \mathrm{G} 418$. Dr Andrew Scott (Ludwig Institute for Cancer Research, Melbourne,

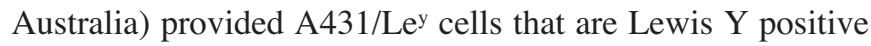
variants of A431. They were cultured in DMEM/F12 supplemented with $10 \%$ v/v FBS, $2 \mathrm{mM}$ gln and pen/strep.

Antibodies and conjugates. HP67.6 (13) and G5/44 (14) are humanized $\mathrm{IgG}_{4}$ antibodies that specifically recognize human CD33 or CD22, respectively. Rituximab (Rituxan ${ }^{\circledR}$, IDEC Pharmaceuticals Corporation and Genentech, South San Francisco, CA) is a chimeric $\mathrm{IgG}_{1-\mathrm{k}}$ antibody that recognizes CD20. MOPC-21 is a monoclonal $\mathrm{IgG}_{1-\kappa}$ mouse antibody with unknown specificity that is commonly used as negative control in immunodetection methods (15).
For FACS analysis, human IgG (huIgG, Zymed, San Francisco, CA) and mouse IgG and FITC-labeled goat antihuIgG (FITC/ $\alpha$-huIgG, Zymed) were used as control antibody and as secondary antibody, respectively. Conjugation of $\mathrm{N}$-acetyl $\gamma$ calicheamicin dimethyl hydrazide (CalichDMH) was done by means of the acid-labile AcBut [4-(4'-acetylphenoxy)butanoic acid] or AcPAc [(3-acetylphenyl)acetic acid] linkers. Acid stabile conjugates were obtained by linking $\mathrm{N}$-acetyl $\gamma$ calicheamicin dimethyl amide (CalichDMA) with an Amide (4-mercapto-4-methylpentanoic acid) linker to the antibodies. The molar ratio of calicheamicin to antibody showed a variation between $2: 1$ and $6: 1 \mathrm{~mol}: \mathrm{mol}$. The chemical procedures for conjugation were published earlier $(11,16)$.

Synthetic macromolecules (FcPEGL and FcPEGB). Human $\mathrm{IgG} F \mathrm{Fc}$ fragments were purchased from Scripps Laboratories (San Diego, CA). Fc fragments were equilibrated in $20 \mathrm{mM}$ $\mathrm{K}_{2} \mathrm{HPO}_{4}$ containing $20 \mathrm{mM} \mathrm{NaCl}$.

The Fc fragments were PEGylated as follows. Twenty mg of Fc was mixed with either $40 \mathrm{mg}$ of linear $20 \mathrm{kDa}$ PEG [N-hydroxysuccinimidyl ester of methoxy poly(ethylene glycol) propionic acid] or $60 \mathrm{mg}$ of branched (10 kDa) ${ }_{2}$ PEG [N-hydroxysuccinimidyl ester of methoxy poly(ethylene glycol)] in $10 \mathrm{mM}$ potassium phosphate buffer $\mathrm{pH}$ 8.0. Both PEG stocks were made in water and used immediately. The reaction was allowed to proceed at $20^{\circ} \mathrm{C}$ for $60 \mathrm{~min}$.

Apparent MW was determined by SDS-PAGE and permeation chromatography. The average MW based on the elution position of the PEGylated Fc is approximately $250 \mathrm{kDa}$ for the branched $(10 \mathrm{kDa})_{2}$ PEG and approximately $300 \mathrm{kDa}$ for the linear $20 \mathrm{kDa}$ PEGylated Fc. SDS-PAGE indicated that the predominant species were Fc:PEG at a molar ratio of $1: 2$ and $1: 3$.

To PEGylate hP67.6, $50 \mathrm{mg}$ of the antibody was mixed with $100 \mathrm{mg}$ PEG [0.5 $\mathrm{ml}$ of $200 \mathrm{mg} / \mathrm{ml}$ of branched $(10 \mathrm{kDa})_{2}$ PEG stock] in $40 \mathrm{mM}$ HEPES buffer $\mathrm{pH} 8.0$, at a final protein 
Table II. Tumor volume reduction of CD33-negative xenografts following treatment with hP67.6-AcBut-CalichDMH.

\begin{tabular}{|c|c|c|c|c|c|c|c|c|c|c|}
\hline \multirow[b]{2}{*}{ Tumor origin } & \multirow[b]{2}{*}{ Cell line } & \multirow[b]{2}{*}{ Expt no. } & \multirow{2}{*}{$\begin{array}{l}\text { Time after } \\
\text { first dose } \\
\text { (days) }\end{array}$} & \multicolumn{7}{|c|}{ Tumor growth inhibition, $\mathrm{I}^{\mathrm{a}}(\%)$} \\
\hline & & & & $0.5^{b}$ & 0.75 & 1 & 1.5 & 2 & 3 & 4 \\
\hline \multirow[t]{6}{*}{ Gastric } & N87 & 1 & 27 & - & - & 45 & - & 59 & - & 81 \\
\hline & & 2 & 27 & - & - & - & - & - & - & 40 \\
\hline & & 3 & 29 & - & - & - & - & 46 & - & 52 \\
\hline & & 4 & 30 & - & - & - & - & - & - & 61 \\
\hline & & 5 & 32 & - & - & - & - & - & - & 58 \\
\hline & & 6 & 32 & - & - & - & - & - & - & 61 \\
\hline \multirow[t]{4}{*}{ Colon } & HT29 & 1 & 32 & - & - & - & - & - & - & 64 \\
\hline & LOVO & 1 & 25 & - & - & - & - & - & - & 20 \\
\hline & & 2 & 29 & - & - & - & - & - & - & 24 \\
\hline & & 3 & 29 & - & - & - & - & - & - & 53 \\
\hline \multirow[t]{6}{*}{ Lung } & L2987 & 1 & 21 & - & 67 & - & 23 & - & 95 & - \\
\hline & & 2 & 29 & - & - & - & - & 95 & - & 99 \\
\hline & & 3 & 34 & - & - & - & - & - & - & 99 \\
\hline & PC14PE6 & 1 & 17 & - & - & - & - & 41 & - & 73 \\
\hline & & 2 & 22 & - & - & - & - & - & - & 85 \\
\hline & & 3 & 29 & - & - & 48 & - & 57 & - & 86 \\
\hline \multirow[t]{2}{*}{ Breast } & MDAMB435/5T4 & 1 & 29 & - & - & - & - & 55 & - & 68 \\
\hline & & 2 & 34 & - & - & - & - & - & - & 70 \\
\hline \multirow[t]{5}{*}{ Cervical } & A 431 & 1 & 27 & - & - & - & - & 28 & - & 79 \\
\hline & & 2 & 27 & - & - & 0 & - & - & - & 65 \\
\hline & & 3 & 28 & - & - & - & - & - & - & 88 \\
\hline & A431/Le & 1 & 21 & - & 38 & - & 61 & - & - & - \\
\hline & & 2 & 29 & - & - & - & - & 62 & - & 99 \\
\hline \multirow[t]{4}{*}{ Prostate } & $\mathrm{LNCaP}$ & 1 & 28 & 0 & - & 41 & - & 70 & - & 76 \\
\hline & & 2 & 29 & - & - & - & - & - & - & 93 \\
\hline & & 3 & 29 & - & - & - & - & 66 & - & - \\
\hline & PC3MM2 & 1 & 29 & - & - & - & - & - & - & 67 \\
\hline
\end{tabular}

${ }^{\mathrm{a} I} \%=100 \mathrm{X}(1-\mathrm{T} / \mathrm{C})$, where $\mathrm{T}$ is the average tumor volume of the hP67.6-AcBut-CalichDMH treated mice at a given time and $\mathrm{C}$ is the average tumor volume of the control mice at the same time. ${ }^{b}$ Nos. in italics indicate the amount of CalichDMH given per dose per mouse. All mice received a total of 3 doses. Treatment was given intraperitoneally at days 1, 5 and 9.

concentration of $10.6 \mathrm{mg} / \mathrm{ml}$. The reaction was allowed to proceed at $20^{\circ} \mathrm{C}$ for $60 \mathrm{~min}$.

Flow cytometry. Aliquots of $10^{5}$ cells were suspended in $100 \mu 1$ phosphate-buffered saline supplemented with $1 \% \mathrm{v} / \mathrm{v}$ bovine serum albumin. The cells were then incubated at $4{ }^{\circ} \mathrm{C}$ for $30 \mathrm{~min}$ in $10 \mu \mathrm{g} / \mathrm{ml}$ primary antibody (hP67.6, G5/44 or rituximab) or conjugates of these antibodies as specified in Results. Binding of the primary antibody to the cells was revealed by FITC/ $\alpha$-huIgG.

Determination of the ED $D_{50}$ of hP67.6-AcBut-CalichDMH and CalichDMH in vitro. A vital dye (MTS) staining was used to determine the number of surviving cells following exposure to various treatments. MTS (non-radioactive cell proliferation assay kit) was purchased from Promega (Madison, WI) and used according to the manufacturer's specifications. For each cell line a calibration curve (cell number versus optical density after $2 \mathrm{~h}$ ) was established to estimate an appropriate initial seeding density. Cells were then seeded in 96-multiwell dishes at a density of 750-5,000 cells per well. Immediately after seeding, the cells were exposed to various concentrations (range 0-500 ng calicheamicin equivalents/ml) of hP67.6AcBut-CalichDMH or CalichDMH. Following determination of the number of cells surviving $96 \mathrm{~h}$ of drug exposure, the $\mathrm{ED}_{50}$ was calculated based on the logistic regression parameters derived from the dose-response curves. The $\mathrm{ED}_{50}$ was defined as the concentration of drug ( $\mathrm{ng} / \mathrm{ml} \mathrm{CalichDMH)}$ that caused a $50 \%$ reduction of the cell number after $96 \mathrm{~h}$.

Efficacy of calicheamicin conjugates in vivo. Subcutaneous tumors of cells specified in Tables I and II were grown in 
athymic nude mice (Charles River, Wilmington, MA). Twomonth-old female mice were injected with respectively $5 \times 10^{6}$ MDA MB435/5T4, HT29, N87, LOVO, A431 or A431/Le cells per mouse. L2987, PC3MM2, LNCaP or PC14PE6 cells were injected in male nude mice that were between 7 and 8 weeks old. To grow tumors, N87, MDAMB435/5T4 and LNCaP cells had to be mixed $\left(1: 1\right.$, vol/vol) with Matrigel ${ }^{\circledR}$ (Collaborative Biomedical Products, Belford, MA) prior to injection. Two perpendicular diameters of the tumor were measured by means of calipers at time intervals specified in the figures. The tumor volume was calculated according to the formula of Attia and Weiss (17): $\mathrm{A}^{2} \mathrm{xBx} 0.4$. A and B are symbols for the smaller and the larger tumor diameter, respectively. The treatment schedules, dose and number of mice per group are specified in Results and in the figure legends. All experiments with living animals were conducted conforming to PRACUC guidelines for humane treatment of animals.

In vivo distribution of ${ }^{125}$ I-labeled conjugate. Gemtuzumab ozogamicin was labeled with ${ }^{125} \mathrm{I}$ using the Bolton-Hunter reagent (NEN, Boston, MA). A group of 30 tumor-bearing female nude mice were injected in the lateral tail vein with ${ }^{125} \mathrm{I}$-labeled conjugate $20 \mu \mathrm{Ci} / 200 \mathrm{mg}$. The tumor weight at the time of injection was approximately $1 \mathrm{~g}$. Groups of 5 mice were sacrificed by $\mathrm{CO}_{2}$ inhalation at 2, 6, 24, 48, 72 and $96 \mathrm{~h}$ following the injection. The amount of $\gamma$-radiation in the tissues as specified in Fig. 2 was determined at these time points. Biodistribution of the conjugate was expressed as a percentage of the injected dose per gram tissue (\% ID/g) or as a percentage of the blood level at a given time point (\% Blood).

\section{Results}

Design of calicheamicin conjugates. Calicheamicin analogues were conjugated to various carrier molecules by either acidlabile or acid-stabile linkers. The acid-labile 4-(4'-acetylphenoxy) butanoic acid (AcBut) or (3-acetylphenyl) acetic acid (AcPAc) linkers allow for acid hydrolysis of the hydrazone group followed by disulfide reduction $(16,18)$. The acidstabile 4-mercapto-4-methyl-pentanoic acid (Amide) linker allows only for dissociation at the disulfide group (18). The calicheamicin analogues, $\mathrm{N}$-acetyl $\gamma$ calicheamicin dimethyl hydrazide (CalichDMH) or $\mathrm{N}$-acetyl $\gamma$ calicheamicin dimethyl acid (CalichDMA) were conjugated with acid-labile or acidstabile linkers, respectively. The various conjugates will be designated as follows: 'carrier-linker-calicheamicin analogue'.

hP67.6-AcBut-CalichDMH inhibits growth of various subcutaneous xenografts despite undetectable amounts of the targeted antigen, CD33, on the tumor cells. The oncolytic effect of hP67.6-AcBut-CalichDMH was demonstrated in multiple xenograft models. Table I lists the carcinoma cell lines used to generate xenografts in nude mice and their expression of CD33 as measured by flow cytometry. The signal obtained using hP67.6 or hP67.6-AcBut-CalichDMH as primary antibody was in most instances similar to the signal obtained after using a negative control antibody, huIgG 4 . The relative median channel fluorescence (reMCF), which is the increase of the median channel fluorescence of a cell population probed with hP67.6 or hP67.6-AcBut-CalichDMH over that of a
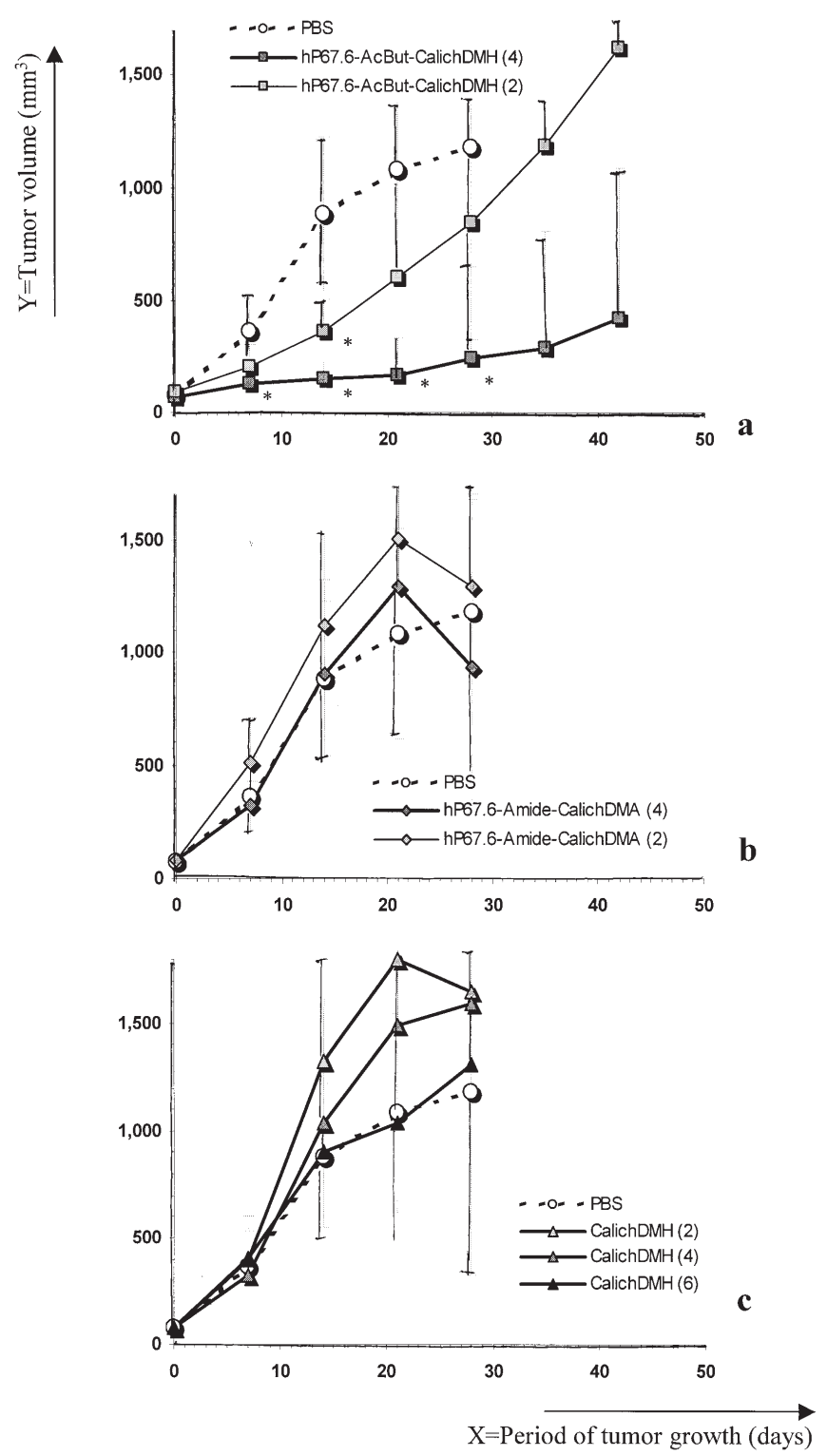

Figure 1. Growth inhibition of CD33-negative epidermoid carcinoma (A431) xenografts by hP67.6-AcBut-CalichDMH. Linking of calicheamicin with an acid-labile AcBut linker to hP67.6 yields an effective tumor inhibiting conjugate (a). Substituting AcBut linker for an acid-stabile Amide linker eliminates the efficacy of the conjugate (b). Administration of free calicheamicin does not cause inhibition of tumor growth (c). Symbols represent the average tumor volumes of 10 (PBS treatment) or 5 animals (calicheamicin or conjugate treatments). Error bars indicate the standard deviation. Mice with xenografts of approximately $80 \mathrm{~mm}^{3}$ received a regimen of 1 dose per mouse, given 3 times intraperitoneally with an interval of 4 days (Q4Dx3). The number between brackets in the figure legends indicates the amount of calicheamicin ( $\mu \mathrm{g} / \mathrm{mouse})$ given in a single dose as free drug or in conjugated form. *Significantly $(\mathrm{p}<0.05)$ different from the vehicle control according to double tailed Student's t-test.

population probed with huIgG $\mathrm{Ig}_{4}$, approximated 1 (maximum 3.1). In comparison, the reMCF of CD33-positive KG-1 myeloid leukemia cells was 9.1.

HP67.6-AcBut-CalichDMH inhibited tumor growth of A431 epidermoid carcinoma xenografts despite the absence of CD33 on the cell suface (Fig. 1). All the groups of mice in the experiment were treated with a single intraperitoneal dose per mouse every 4 days for a period of 9 days (Q4Dx3). Mice with xenografts of approximately $80 \mathrm{~mm}^{3}$ were treated. The 
Tumor

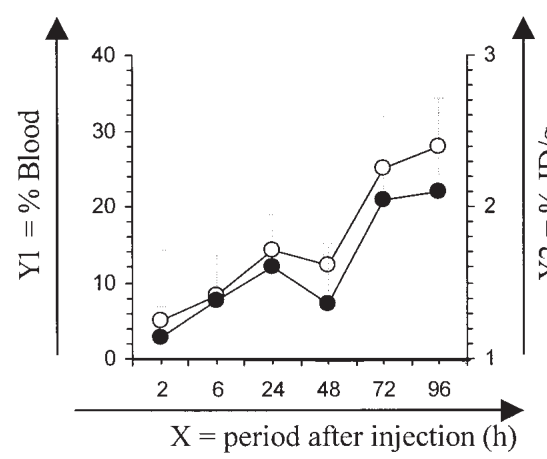

Brain

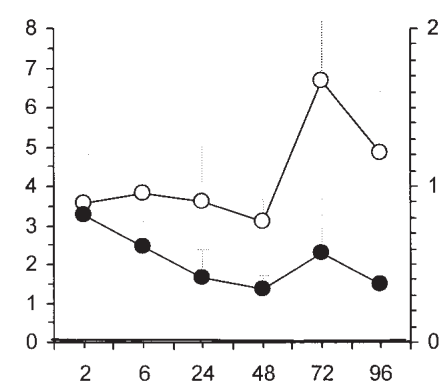

Striated muscle

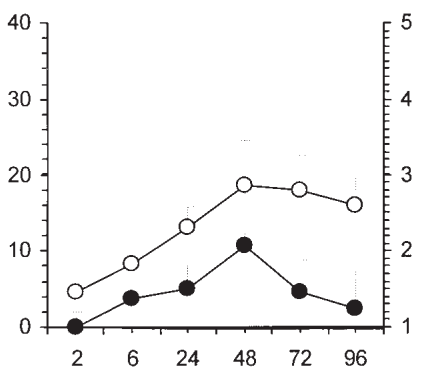

Blood

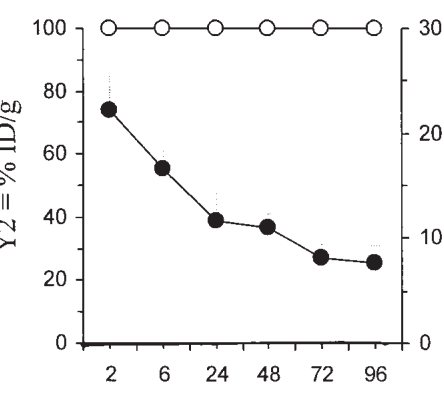

Skin

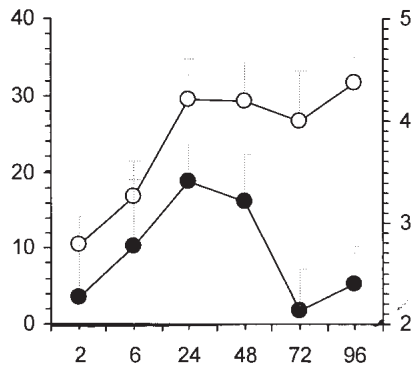

Lung

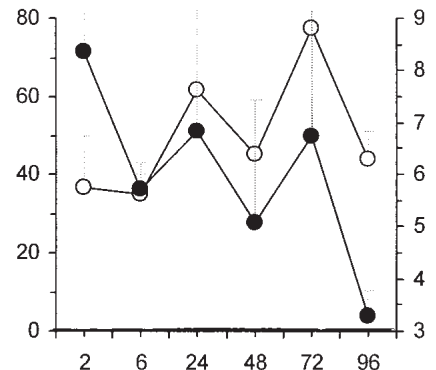

Heart

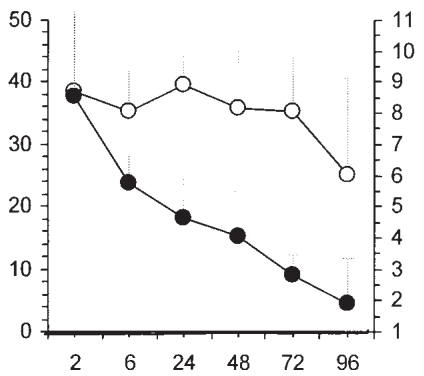

Liver

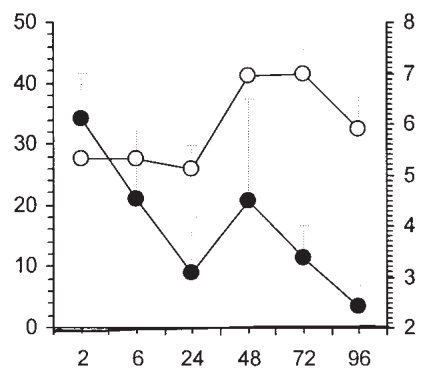

Spleen

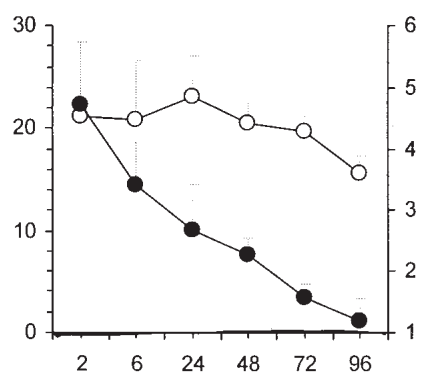

Kidney

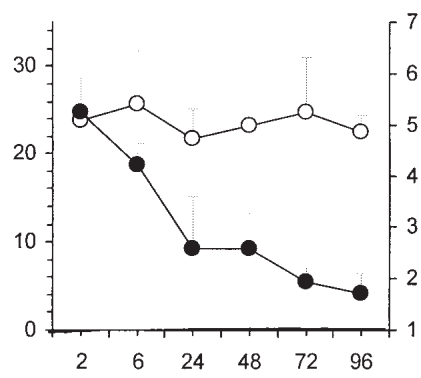

Intestine

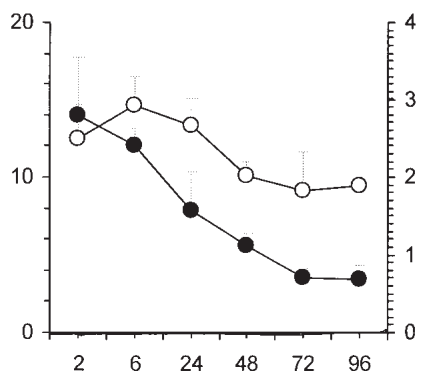

Figure 2. Distribution of ${ }^{125} \mathrm{I}$-labeled hP67.6-AcBut-CalichDMH in function of time in CD33-negative tumor-bearing mice. The amounts of hP67.6-AcButCalichDMH in the various normal tissues and tumor (A431) xenografts are presented relative to the amount of conjugate in total blood (open circles, Y1 axis, $\%$ Blood) or to the amount injected (closed circles, Y2 axis, \% ID/g). All data points reflect the mean of 5 samples. Error bars indicate the standard deviations. Steadily increasing concentrations of hP67.6-AcBut-CalichDMH were exclusively observed in tumor tissue. The doubling time of accumulation in A431tumors is $151 \mathrm{~h}$.

growth of A431 xenografts was significantly $(\mathrm{p}<0.05)$ inhibited following administration of 3 doses of $4 \mu \mathrm{g}$ hP67.6-AcButCalichDMH for up to 27 days after treatment. Xenografts treated with $2 \mu \mathrm{g}$ /dose hP67.6-AcBut-CalichDMH were significantly $(\mathrm{p}<0.05)$ smaller than the controls at day 13 following the first injection (Fig. 1a).
Administration of hP67.6-Amide-CalichDMA or Calich DMH at equivalent or higher doses than hP67.6-AcButCalichDMH did not inhibit tumor growth (Fig. 1b and c). The results in Fig. 1 demonstrated not only a significant inhibition of tumor growth by hP67.6-AcBut-CalichDMH but also dependence of this effect on the linker used for conjugation. 
The ineffectiveness of CalichDMH indicates that release of calicheamicin from the conjugate in plasma is unlikely to cause the growth inhibition. To exclude the possibility that the efficacy of hP67.6-AcBut-CalichDMH was caused by slow release of CalichDMH from the peritoneum, the experiment was repeated using the intravenous route of administration of the drugs while maintaining the same dose, frequency and interval of the treatments. Significant growth inhibition was observed following treatment with hP67.6-AcBut-CalichDMH. Twenty-seven days following onset of therapy, the average tumor growth inhibition of mice treated with 2 or $4 \mu \mathrm{g} /$ dose of this conjugate were 77 or $89 \%$, respectively. Tumor growth inhibition $[\mathrm{I} \%=100 \mathrm{X}(1-\mathrm{T} / \mathrm{C})]$ reflects reduction of the volume of treated tumors compared to controls. $\mathrm{T}$ stands for the average tumor volume of a group of mice that were treated with hP67.6-AcBut-CalichDMH and C is the average tumor volume of a control group. Both $\mathrm{T}$ and $\mathrm{C}$ were determined on the same day after treatment initiation. Intravenous administration of hP67.6-Amide-CalichDMA or CalichDMH did not yield significant tumor growth inhibition.

HP67.6-AcBut-CalichDMH inhibited tumor growth of human tumor xenografts from diverse histotypic origin (Table II). The I \%-values were derived from 27 independent experiments and were determined between 17 and 34 days after injection of the first dose of hP67.6-AcBut-CalichDMH. Despite variability in magnitude of the response, the data demonstrate that hP67.6-AcBut-CalichDMH at a dose of $4 \mu \mathrm{g} /$ mouse (Q4Dx3) inhibits tumor growth in the majority of xenografts. Inhibition was also observed when lower amounts of the conjugate were administered.

${ }^{125}$ I-labeled hP67.6-AcBut-CalichDMH conjugate accumulates in CD33-negative A431-tumor xenografts. The kinetics of hP67.6-AcBut-CalichDMH in various mouse tissues and xenografted tumor were compared. Following injection of $200 \mu \mathrm{g}$ $(20 \mu \mathrm{Ci})$ of ${ }^{125} \mathrm{I}$-labeled conjugate, the amount of radioactive label was measured in various tissues at 2, 6, 24, 48, 72 and $96 \mathrm{~h}$ (Fig. 2). This amount was expressed relative to the amount present in whole blood at the time of measurement $(\%$ Blood $=$ $100 \times \mathrm{Bq}$ per gram tissue/ $\mathrm{Bq}$ per gram blood) or relative to the total amount of conjugate given (\% ID/g). Because of the blood-brain barrier, accumulation of the conjugate in the brain was not anticipated. Indeed, only a marginal amount of ${ }^{125} \mathrm{I}-$ labeled conjugate was retained in the brain $(\%$ Blood $=3.5 \%)$ and this did not significantly vary within $96 \mathrm{~h}$.

During a period of $96 \mathrm{~h}$, the amount of hP67.6-AcButCalichDMH in tumor tissue relative to the amount in whole blood increased from 6 to $28 \%$. This steady increase was exclusively found in tumor tissue. The \% Blood-values of heart, intestine and spleen were highest at $2 \mathrm{~h}$ after injection and then steadily decreased with time. In liver and striated muscle, the peak of the \% Blood-value was at $48 \mathrm{~h}$. In skin, this value reached a plateau after $24 \mathrm{~h}$. The increase of the \% Bloodvalue in tumor tissue was not solely the indirect result of clearance of hP67.6-AcBut-CalichDMH from the blood but was also due to an increase of antibody concentration in tumor tissue. This was evidenced by a steady increase (doubling time $=151 \mathrm{~h}$ ) of the $\% \mathrm{ID} / \mathrm{g}$ value, which indicates the absolute amount of hP67.6-AcBut-CalichDMH in the tissue. In contrast to tumor-tissue, the $\% \mathrm{ID} / \mathrm{g}$ decreased over time in all of
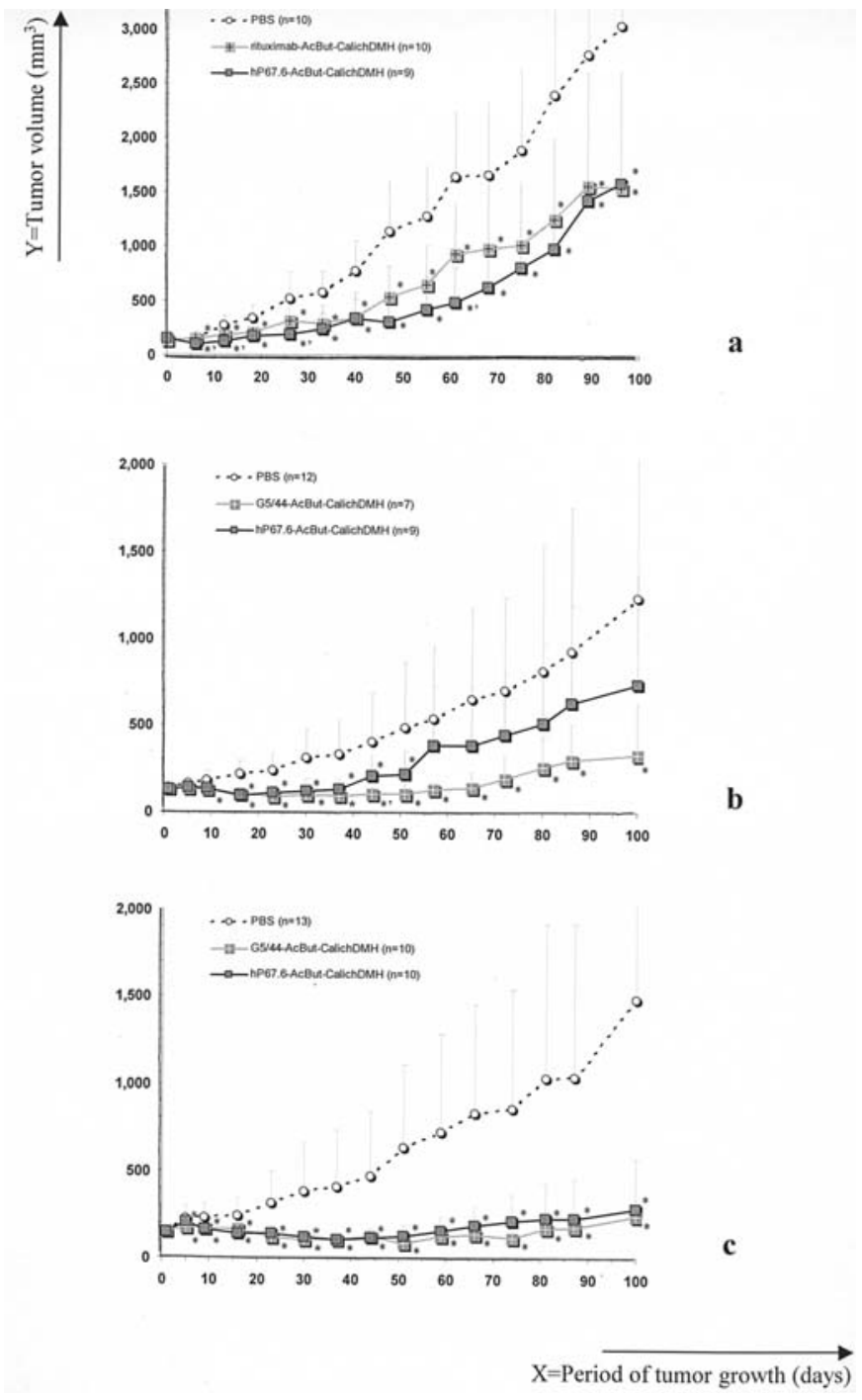

Figure 3. Passive targeting of calicheamicin using hP67.6, G5/44 and rituximab as carriers inhibits tumor growth. The influence of hP67.6-AcBut-CalichDMH on growth of $\mathrm{N} 87$ xenografts was compared to that of rituximab-AcButCalichDMH (a). Efficacy of G5/44-AcBut-CalichDMH and hP67.6-AcButCalichDMH against N87 (b) or MDAMB435/5T4 (c) is also demonstrated. All the groups of mice treated with conjugate received a regimen of 1 dose of $4 \mu \mathrm{g}$ CalichDMH per mouse, given 3 times intraperitoneally with an interval of 4 days (Q4Dx3). Each point represents the average of $n$ tumor measurements (see legend). Error bars reflect the standard deviation. ${ }^{*}$ Significantly $(\mathrm{p}<0.05$, double tailed Student's t-test) different from the vehicle control. 'Significantly $(\mathrm{p}<0.05$, double tailed Student's t-test) different from the treatment with the other calicheamicin conjugate.

the other tissues that we examined. Tumor tissue was thus exceptional in its capacity to retain and accumulate hP67.6AcBut-CalichDMH. The ${ }^{125}$ I-label indicated accumulation of the antibody in tumor tissue but did not demonstrate whether the CalichDMH part of the conjugate follows a similar accumulation trend. The tissue distribution of hP67.6 conjugated to ${ }^{3} \mathrm{H}$-labeled CalichDMH (Dr Joseph McDevitt, personal communication) was similar to that of ${ }^{125}$ I-labeled conjugate. Thus, the cytotoxic component of the conjugate was similarly distributed as the immunoglobulin carrier in both normal and neoplastic tissues.

Calicheamicin conjugates of rituximab and G5/44 inhibit tumor growth to the same extent as hP67.6-AcBut-CalichDMH. 

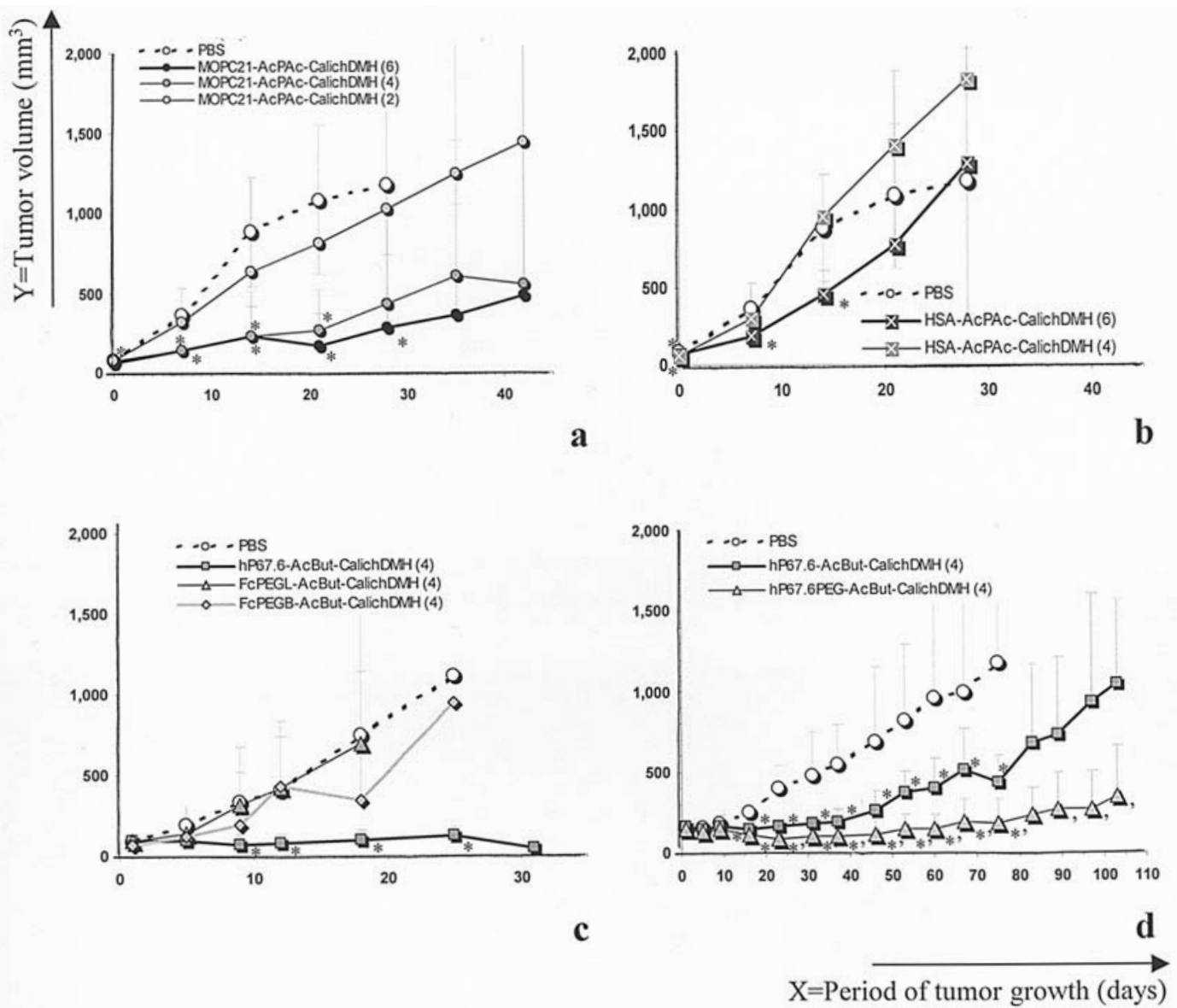

Figure 4. Tumor growth inhibition by calicheamicin conjugates of HSA, PEGylated Fc and PEGylated hP67.6. The influence of MOPC-21-AcPAcCalichDMH (a) on growth of A431 xenografts was compared to that of HSA-AcPAc-CalichDMH (b). Each point represents the average tumor volume of 5 (conjugate treatments) or 10 (PBS) xenografts. All the groups of mice received a regimen of 1 dose per mouse, given 3 times intraperitoneally with an interval of 4 days (Q4Dx3). The number between brackets in the figure legends indicates the amount of calicheamicin $(\mu \mathrm{g} / \mathrm{mouse})$ given in a single dose. In panel (c) the inhibition of A431 xenograft growth by calicheamicin conjugates of PEGylated Fc fragments was compared to that of hP67.6-AcBut-CalichDMH. Each point represents the average tumor volume of 5 (conjugate treatments) or 10 (PBS) xenografts. The efficacy of calicheamicin conjugates of hP67.6 and the PEGylated form of the antibody (hP67.6PEGB) was tested against N87 tumor xenografts (d). Each point represents the average of tumor volumes for groups of 10 mice treated with PBS or hP67.6PEGB-AcBut-CalichDMH and the average of 7 for the group of mice treated with hP67.6-AcBut-CalichDMH. Concerning the experiments presented in panels (c) and (d), all the groups of mice treated with conjugate received a regimen of 1 dose of $4 \mu \mathrm{g}$ CalichDMH per mouse, given 3 times intraperitoneally with an interval of 4 days (Q4Dx3). Error bars in all panels reflect the standard deviation. 'Significantly ( $<<0.05$, double tailed Student's t-test) different from the treatment with the other calicheamicin conjugate.

To verify whether tumor growth inhibition caused by hP67.6AcBut-CalichDMH was restricted to hP67.6 as a carrier for passive targeting, we compared the efficacy of the hP67.6 conjugate to that of rituximab and G5/44 conjugates. HP67.6 and $\mathrm{G5} / 44$ are humanized $\mathrm{IgG}_{4}$ molecules. Rituximab is a mouse-human $\mathrm{IgG}_{1}$ chimera. G5/44 and rituximab recognize CD22 and CD20, respectively. None of the 3 antibodies bound with high avidity to N87 or MDAMB435/5T4. ReMCF values for hP67.6 are listed in Table I. The average reMCF values after probing N87 or MDAMB435/5T4 with rituximab were 1.41 and 0.89 , respectively. After probing these cells with $\mathrm{G} 5 / 44$, the average reMCF values were respectively 0.76 and 1.85. Despite the poor binding of the antibodies to the cell lines, their calicheamicin conjugates caused significant $(p<0.05)$ inhibition of tumor growth (Fig. 3). The quantitative differences in efficacy among the various conjugates were rarely statistically significant (Fig. 3 legend). Equivalent efficacy was thus achieved with the conjugates regardless of the specificity or isotype variant of the antibody used for conjugation.
Substituting the immunoglobulin by either human serum albumin (HSA) or PEGylated Fc fragments reduces the efficacy of calicheamicin conjugates. The data presented in Fig. 4 indicated that for a calicheamicin conjugate to retain optimal efficacy, neither human serum albumin nor PEGylated Fc could replace the carrier antibody. Fig. 4a shows the growth inhibition by the MOPC-21-AcPAc-CalichDMH conjugate. MOPC-21 (15) is a mouse monoclonal antibody $\left(\operatorname{IgG}_{1}\right)$ with unknown specificity that is commonly used as negative control in immunodetection methods. The AcPAc linker was used to conjugate calicheamicin to this antibody. This conjugate's efficacy indicated that using the AcPAc linker did not prevent oncolytic effects of the conjugate. In comparison, the efficacy of HSA-AcPAc-CalichDMH was marginal within the same experiment (Fig. 4b). Although this conjugate was more efficacious in another experiment ( $\mathrm{I}=61 \%$ at 20 days after administration of $4 \mu \mathrm{g}$ /mouse Q4Dx3), it did not have higher efficacy than the control antibody conjugate ( $\mathrm{I}=77 \%)$. The requirement to use a complete immunoglobulin was further illustrated in Fig. 4c. In this experiment, we compared tumor 
growth inhibition by hP67.6-AcBut-CalichDMH to the efficacy of a conjugate consisting of a PEGylated Fc fragment linked to calicheamicin by an AcBut linker. Two types of PEG were used to increase the Stokes' radius of the conjugate. The FcPEGL (apparent MW = $300 \mathrm{kDa}$ ) was PEGylated with the linear N-hydroxysuccinimidyl ester of methoxy poly(ethylene glycol)propionic acid. FcPEGB was PEGylated with a branched form of this molecule (apparent $\mathrm{MW}=250 \mathrm{kDa}$ ). Regardless of the nature of the PEG, the Fc conjugates failed to cause any growth inhibition. Alternatively, a complete PEGylated (branched PEG) antibody (hP67.6PEGB, apparent $\mathrm{MW}=300 \mathrm{kDa}$ ) conjugated to calicheamicin inhibited tumor growth more than hP67.6-AcBut-CalichDMH, indicating that PEGylation per se did not abrogate the efficacy of a conjugate (Fig. 4d).

The efficacy caused by passive targeting of hP67.6-AcButCalichDMH correlates with the sensitivity of tumor cells to calicheamicin in vitro. The sensitivity of 10 tumor cell lines to CalichDMH and to hP67.6-AcBut-CalichDMH was tested in vitro. The $\mathrm{ED}_{50}$ of these two drugs was defined as the lowest concentration $(\mathrm{ng} / \mathrm{ml})$ that reduced the number of cells in a monolayer after $96 \mathrm{~h}$ to $50 \%$ of an untreated control culture. The rank order of the various cell lines was similar whether $\mathrm{ED}_{50}$ of CalichDMH or $\mathrm{ED}_{50}$ of hP67.6-AcButCalichDMH was used as a ranking criterion (compare Fig. 5a with $\mathrm{b}$ ). The linear regression coefficient of $\mathrm{ED}_{50}$ of hP67.6AcBut-CalichDMH as a function of $\mathrm{ED}_{50}$ of CalichDMH was 0.79. The sensitivity of the subcutaneous xenografts to hP67.6AcBut-CalichDMH was reflected by the maximum tumor growth inhibition $\left(\mathrm{I}_{\max } \%\right)$. This parameter is the maximum I-value observed during a given experiment. Hence, the $I_{\max }$ allowed a comparison of the efficacy of hP67.6-AcButCalichDMH on the various xenografts. Fig. 5 demonstrates that $I_{\max }$ of these xenografts was inversely proportional to the $\mathrm{ED}_{50} \mathrm{~s}$ determined after addition of CalichDMH ( $\mathrm{r}=0.41$, Fig. 5a) or hP67.6-AcBut-CalichDMH (r=0.63, Fig. 5b) to the cells from which the tumors originated. This crude correlation suggested that sensitivity to CalichDMH was a determinant for the efficacy of hP67.6-AcBut-CalichDMH. However, the exceptionally low $\mathrm{I}_{\max }$ found for LOVO colon carcinoma implied that sensitivity to CalichDMH alone was not sufficient to explain efficacy by passive targeting.

\section{Discussion}

The presented results show that calicheamicin-immunoglobulin conjugates cause tumor regression in various human tumor xenografts. These tumors did not display detectable amounts of antigen recognized by the immunoglobulin. Therefore, passive targeting of calicheamicin by means of an immunoglobulin carrier is a potential strategy to safely administer a therapeutically effective amount of calicheamicin.

By substituting immunoglobulin of the conjugate with other macromolecules, we identified carrier characteristics underlying the therapeutic activity of a CIgC. Consistent with the finding that accumulation of immunoglobulin in grafted tumors was more pronounced than the accumulation of albumin (9), we found that the antibody could neither be

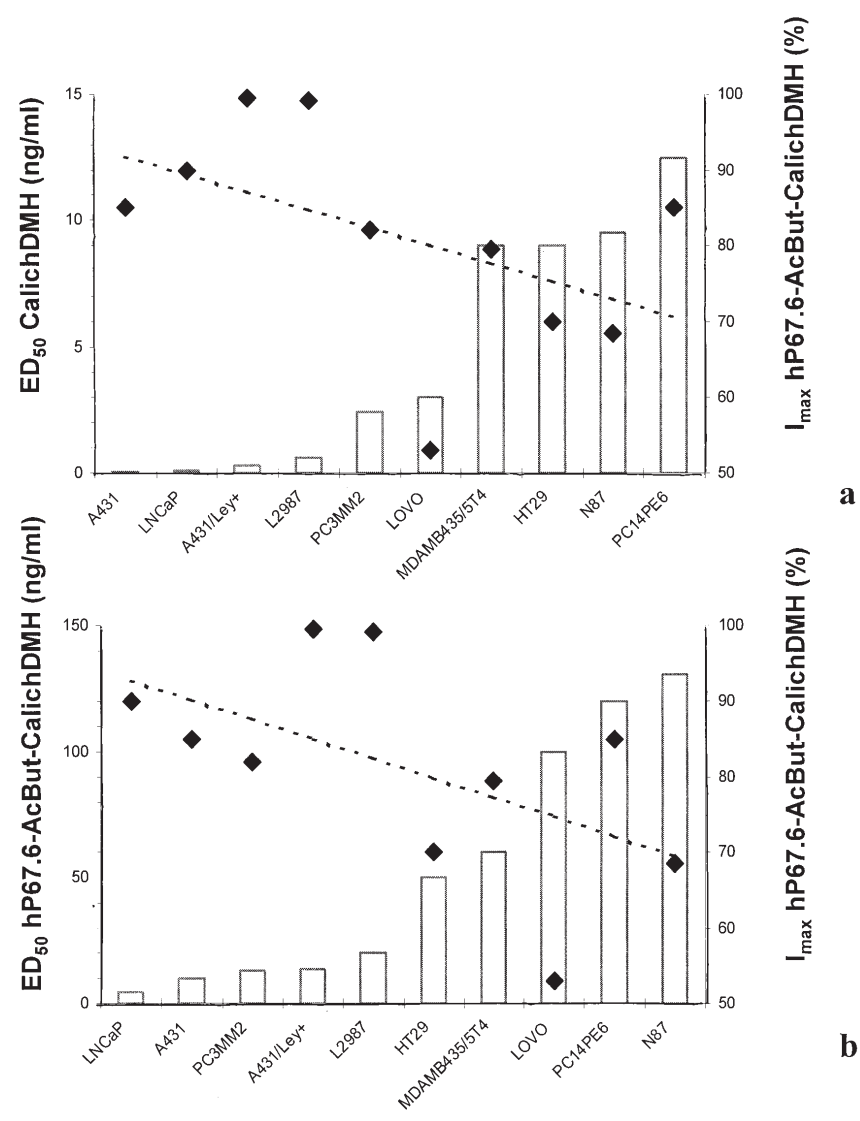

Figure 5. Inhibition of tumor growth by passive targeting of calicheamicin correlates with sensitivity of the tumor cells to calicheamicin in vitro. The sensitivity of tumor cell lines [X axes, (a) and (b)] to calicheamicin is presented as $\mathrm{ED}_{50^{-}}$-value of either CalichDMH [Y1 axis, (a)] or hP67.6AcBut-CalichDMH [Y1 axis, (b)]. The height of each bar reflects the median of at least 3 independent $\mathrm{ED}_{50}$ determinations. The sensitivity of the tumor xenografts to hP67.6-AcBut-CalichDMH is expressed as $I_{\max }$ [Y2 axes, (a) and (b)]. The $I_{\max }$ values (black diamonds, dashed linear regression curve) are determinations obtained from either a single experiment (A431/Le ${ }^{\mathrm{y}}, \mathrm{PC} 3 \mathrm{MM} 2$ and HT29) or the median of multiple experiments [N87 $(n=6)$, PC14PE6 $(n=3)$, LOVO (n=3), L2987 ( $n=2)$, MDAMB435/5T4 $(n=2)$, A431 $(n=3)$, LNCaP $(n=2)]$. All the $I_{\max }$ values for hP67.6-AcBut-CalichDMH were determined following treatment with a regimen of 1 dose of $4 \mu \mathrm{g}$ CalichDMH per mouse, given 3 times intraperitoneally with an interval of 4 days (Q4Dx3).

replaced by albumin nor by PEGylated Fc fragments without reducing efficacy of the conjugate. When administered Q4Dx3, calicheamicin conjugates of human $\operatorname{IgG}_{1}$ (rituximab), human $\mathrm{IgG}_{4}$ (hP67.6, G5/44) or mouse $\mathrm{IgG}_{1}$ had similar efficacy. Thus, molecular weight and protein structure of the $\mathrm{IgG}$ molecule were necessary to target sufficient amounts of calicheamicin to the tumor without causing lethality in the mice. Since the isoelectric point of the carrier antibodies ranged from $\mathrm{pH} 6.0$ (MOPC-21) to 9.0 (rituximab), our experiments did not substantiate an earlier prediction that the negative charge of the molecule would be a major determinant for its accumulation (9).

Apart from the carrier function of the immunoglobulin, the use of an acid-labile linker (18) proved crucial for the efficacy of $\mathrm{CIgC}$. This indicated that after accumulation of $\mathrm{CIgC}$ in the tumor, the pericellular acidic environment (19) was responsible for the release of calicheamicin. This mechanism was further inferred from the congruence of oncolytic effects 
by $\mathrm{CIgC}$ in vivo with the sensitivity of tumor cells to calicheamicin in vitro. We cannot exclude a contribution of pinocytosis as a mechanism for intracellular uptake of the $\mathrm{CIgC}$. However, the observation that a CIgC constructed with an acid-stabile linker was ineffective in the absence of a targeted antigen renders the contribution of this mechanism less crucial.

We showed previously (11) that active targeting of calicheamicin to $\mathrm{Le}^{\mathrm{y}}$ was more efficacious than passive targeting. This manifested itself by shorter duration of the tumor remission and the higher doses necessary to obtain efficacy with a $\mathrm{CIgC}$ that uses a passive targeting mechanism. Yet, a passive targeting strategy may in certain circumstances be indicated because it bypasses the need for homogeneous (over)expression of a TAA. Furthermore, TAAs are rarely exclusive products of tumor cells. Expression of TAAs [e.g. Le $^{y}$ (20), EGFR (21), Her2/neu (22)] in normal tissues can pose therapeutic dose limiting toxicity for $\mathrm{CIgC}$ that recognize these antigens.

A CIgC with a carrier antibody that does not recognize any human antigen could theoretically bypass this problem. It should also be noted that comparisons between active and passive targeting with $\mathrm{CIgC}$ have been based on dose and regimen equivalence indicated by the amount of calicheamicin $(11,16)$ and not on the maximum tolerated dose. Theoretically, passive targeting may yield similar therapeutic indices as CIgCs that target TAAs because a reduced accumulation in the tumor could be compensated by a higher maximum tolerated dose. Unfortunately, this hypothesis can only be tested in the clinic since these $\mathrm{CIgCs}$ do not always recognize the mouse homologues of the targeted antigen.

Thus far, clinical trials that can determine efficacy of a $\mathrm{CIgC}$ designed for passive targeting have not been conducted. Nonetheless, the potential validity of this approach can be deduced from reports that show either accumulation of nonspecific IgG in tumors (10) or therapeutic response of a CIgC, $\mathrm{GO}$, in patients whose cancer cells do not display the targeted antigen, CD33 (23). Pancreatic tumors and liver metastases can be visualized by single photon emission computed tomography using non-specific IgG labeled with ${ }^{111}$ In (10). These clinical observations refute the notion that accumulation of 'non-specific' $\mathrm{IgG}$ in a tumor would be an artifact created in xenografts. The therapeutic responses to GO by CD33- AML patients have been explained by endocytosis of the conjugate by malignant cells (23), 'background' levels of CD33 (24) or the presence of a small subpopulation of CD33+ leukemic progenitors (24). Because AML presents itself as a neovascularized tumor mass in bone marrow (25), we propose a mechanism of passive targeting of the conjugate based on the EPR effect.

In conclusion, we have demonstrated that passive targeting of CIgCs yields therapeutic benefit in a variety of human tumor xenografts. We have also shown that characteristics of the IgG molecule and the use of an acid-labile linker were of quintessential importance to allow efficacy by passive targeting. CIgCs designed for passive targeting may prove to be valuable clinical assets in targeted delivery when tumors do not express tumor associated antigen or when extratumoral expression of these antigens prevents the use of actively targeted CIgC.

\section{References}

1. Minko T, Dharap SS, Pakunlu RI and Wang Y: Molecular targeting of drug delivery systems to cancer. Curr Drug Targets 5: 389-406, 2004.

2. Matsumura Y and Maeda HA: A new concept for macromolecular therapeutics in cancer chemotherapy; mechanism of tumoritropic accumulation of proteins and the antitumor agent SMANCS. Cancer Res 6: 6387-6392, 1986.

3. Metselaar JM, Mastrobattista E and Storm G: Liposomes for intravenous drug targeting: design and application. Mini Rev Medicinal Chem 2: 319-329, 2002.

4. Paal K, Muller J and Hegedus L: High affinity binding of paclitaxel to human serum albumin. Eur J Biochem 268: 2187-2191, 2001.

5. Tabata Y, Noda Y, Matsui Y and Ikada Y: Targeting of tumor necrosis factor to tumor by use of dextran and metal coordination. J Controlled Release 59: 187-196, 1999.

6. Allen TM and Cullis P: Drug delivery systems entering the mainstream. Science 303: 1818-1822, 2004.

7. Bross PF, Beitz J, Chen G, et al: Gemtuzumab ozogamicin: approval summary: gemtuzumab ozogamicin in relapsed acute myeloid leukemia. Clin Cancer Res 7: 1490-1496, 2001.

8. Zein N, Sinha AM, McGahren WS and Ellestad GA: Calicheamicin $\gamma_{1}$ : an antitumor antibiotic that cleaves double-stranded DNA site specifically. Science 240: 1198-1201, 1988.

9. Takakura Y, Fujita T, Hashida MW and Sezaki H: Disposition characteristics of macromolecules in tumor-bearing mice. Pharm Res 7: 339-346, 1990.

10. Karanikas G, Ulrich-Pur H, Becherer A, Wiesner K, Dudczak R, Raderer $\mathrm{M}$ and Kletter K: Uptake of indium-111-labeled human polyclonal immunoglobulin $\mathrm{G}$ in pancreatic cancer: in vivo and in vitro studies. Oncol Rep 9: 353-357, 2002.

11. Boghaert ER, Sridharan L, Armellino DC, et al: Antibodytargeted chemotherapy with the calicheamicin conjugate hu3S193$\mathrm{N}$-acetyl $\gamma$ calicheamicin dimethyl hydrazide targets Lewis $\mathrm{Y}$ and eliminates LewisY-positive human carcinoma cells and xenografts. Clin Cancer Res 10: 4538-4549, 2004.

12. Yano S, Nokihara H, Hanibushi M, Parajuli P, Shinohara T, Kawano T and Sone S: Model of malignant pleural effusion of human lung adenocarcinoma in SCID mice. Oncol Res 9: 573-579, 1997.

13. Hamann P, Hinman L, Hollander I, et al: Gemtuzumab ozogamicin, a potent and selective anti-CD33 antibody-calicheamicin conjugate for treatment of acute myeloid leukemia. Bioconjug Chem 13: 47-58, 2002.

14. DiJoseph JF, Armellino DC, Boghaert ER, et al: Antibodytargeted chemotherapy with CMC-544: a CD22-targeted immunoconjugate of calicheamicin for the treatment B lymphoid malignancies. Blood 103: 1807-1814, 2004.

15. Melchers P: Biosynthesis of carbohydrate portion of immunoglobulins. Biochem J 119: 765-772, 1970.

16. DiJoseph JF, Popplewell A, Tickle S, et al: Antibody-targeted chemotherapy of B-cell lymphoma using calicheamicin conjugated to murine or humanized antibody against CD22. Cancer Immunol Immunother 54: 11-24, 2005.

17. Attia MA and Weiss DW: Immunology of spontaneous mammary carcinomas in mice. V. Acquired tumor resistance and enhancement in strain-A mice infected with mammary tumor virus. Cancer Res 26: 1787-1800, 1966.

18. Hamann P, Hinman L, Beyer C, Lindh D, Upeslacis J, Flowers DA and Berstein I: An anti-CD33 antibody-calicheamicin conjugate for treatment of acute myeloid leukemia. Choice of linker. Bioconjug Chem 13: 40-46, 2002.

19. Schomack PA and Gillies RJ: Contributions of cell metabolism and $\mathrm{H}^{+}$diffusion to the acidic $\mathrm{pH}$ of tumors. Neoplasia 5: 135-145, 2003.

20. Sakamoto J, Furakawa K, Cordon-Cardo C, et al: Expression of Lewis a, Lewis b, X, and Y blood group antigens in human colonic tumors and normal tissues and in human tumor-derived cell lines. Cancer Res 46: 1553-1561, 1986.

21. Starling M and Cunningham D: Monoclonal antibodies against vascular endothelial growth factor and epidermal growth factor receptor in advanced colorectal cancers: present and future directions. Curr Opin Oncol 16: 385-390, 2004.

22. Pai-Scherf LH, Villa J, Pearson D, et al: Hepatotoxicity in cancer patients receiving erb-38, a recombinant immunotoxin that targets the erbB2 receptor. Clin Cancer Res 5: 2311-2315, 1999. 
23. Jedema I, Barge RMY, van der Velden VHJ, Nijmeijer BA, van Dongen JJM, Willemze R and Falkenburg JHF: Internalization and cell cycle-dependent killing of leukemic cells by Gemtuzumab Ozogamicin: rationale for efficacy in CD33negative malignancies with endocytic capacity. Leukemia 18: 316-325, 2004.

24. Linenberger ML: CD33-directed therapy with gemtuzumab ozogamicin in acute myeloid leukemia: progress in understanding cytotoxicity and potential mechanisms of drug resistance. Leukemia 19: 176-182, 2005.

25. Padro T, Ruiz S, Bieker R, et al: Increased angiogenesis in the bone marrow of patients with acute myeloid leukemia. Blood 95: 2637-2644, 2000. 